\title{
Comunicación
}

\section{Respuesta a la quimoterapia contra linfoma mediastínico en un gato doméstico}

\author{
Response to chemotherapy against mediastinal lymphoma in a domestic cat
}

Elizabeth Hinostroza M. ${ }^{1,2}$, Ricardo Grandez R. ${ }^{1}$, Sergio Salgado NdP. ${ }^{1}$, Elia Salas C. ${ }^{1}$

\section{Resumen}

Se reporta el tratamiento de un caso de un felino doméstico macho castrado de 2 años y $6.8 \mathrm{~kg}$ de peso vivo con linfoma mediastínico. Al examen clínico mostró temperatura de $38{ }^{\circ} \mathrm{C}$, disnea inspiratoria restrictiva superficial, tiempo de llenado capilar $<2 \mathrm{~s}$, frecuencia cardiaca de $140 \mathrm{lat} / \mathrm{min}$, frecuencia respiratoria 62/min. En los análisis de laboratorio se encontró leucopenia con linfopenia y positivo al virus de la leucemia felina. En la radiografía y ecografía torácica se encontró presencia de efusión pleural moderada y una estructura redondeada hipoecoica de bordes irregulares, respectivamente. La toracocentesis reveló presencia de quilotorax y mediante la citología por punción de aguja fina de la estructura redondeada se dio como diagnóstico final linfoma mediastínico. Se instauró una quimioterapia con L-asparaginasa, vincristina, ciclofosfamida, doxorrubicina y prednisolona. El paciente sobrellevó ocho sesiones de quimioterapia hasta que por reacciones de toxicidad se decidió suspenderlas. Durante el tiempo de tratamiento se logró una remisión parcial del tumor, disminución significativa de la efusión y mejora de la calidad de vida del paciente. El principal efecto adverso fue la mielosupresión. Se logró un tiempo de sobrevida aproximado de más de 112 días.

Palabras clave: virus de leucemia felina; linfoma mediastinal; quimioterapia

\section{AbSTRACT}

It is reported the treatment of a 2-year-old male castrated domestic feline, $6.8 \mathrm{~kg}$ of body weight, with mediastinal lymphoma. At clinical examination, it showed a temperature of $38^{\circ} \mathrm{C}$, superficial restrictive inspiratory dyspnea, $<2$ s capillary refill time, heart rate of 140 beats/min, respiratory rate $62 / \mathrm{min}$. In the laboratory analyzes was found leukopenia

\footnotetext{
${ }^{1}$ Clínica Veterinaria Cayetano Heredia, Facultad de Medicina Veterinaria y Zootecnia, Universidad Peruana Cayetano Heredia, Lima, Perú

${ }^{2}$ E. mail: clarisa.hinostroza@upch.pe
} 
with lymphopenia and positive for the feline leukemia virus. Radiography and thoracic ultrasound showed moderate pleural effusion and a rounded hypoechoic structure with irregular edges, respectively. The thoracocentesis revealed the presence of chylothorax and the cytology by fine needle puncture of the rounded structure allowed a final diagnosis of mediastinal lymphoma. A chemotherapy was instituted with L-asparaginase, vincristine, cyclophosphamide, doxorubicin, and prednisolone. The patient underwent eight chemotherapy sessions until, due to toxicity reactions, it was decided to suspend them. During the treatment period, a partial remission of the tumor, a significant decrease in the effusion and an improvement in the quality of life of the patient were achieved. The main adverse effect was myelosuppression. An approximate survival time of more than 112 days was reached.

Key words: feline leukemia virus; mediastinal lymphoma; chemotherapy

\section{INTRODUCCIÓN}

El linfoma (linfoma maligno o linfosarcoma) es la neoplasia más común del sistema hematopoyético en los gatos domésticos (Louwerens et al., 2005). La forma mediastínica involucra al timo y los nódulos linfáticos esternales y mediastinales; aunque existen reportes de la presentación extranodal, principalmente en tracto digestivo y otros lugares anatómicos como la cavidad nasal, el sistema nervioso central, la piel, los riñones e incluso el útero (Azakami et al., 2015; Kyoung-Won et al., 2006).

Se ha reportado que $73 \%$ de los gatos con linfoma mediastínico están infectados por el virus de la leucemia felina (ViLeF), y que los gatos jóvenes tienen mayor predisposición a la enfermedad; asimismo, se ha determinado una mayor proporción de machos afectados que en hembras (1.5:1) (Vail et al., 1998; Kyoung-Won et al., 2006) y que la raza Siamés parece ser una de las más afectadas aunque sea negativo al ViLeF o al virus de la inmunodeficiencia felina (VIF), debido a que en esta raza existe una forma heredable de esta enfermedad (Kyoung-Won et al, 2006).

Los signos clínicos que presentan los pacientes con linfoma mediastínico son: disnea, taquipnea, disfagia y efusión pleural, sien- do común el observarse en esta última la presencia de linfoblastos. También se han descrito ruidos pulmonares y cardiacos apagados, con percusión mate, siendo menos común la presencia del síndrome de Horner y el edema de cabeza (Ettinger, 2003; KyoungWon et al., 2006).

Para el tratamiento del linfoma se han propuesto diversos esquemas. Moore et al. (1996), utilizando vincristina, ciclofosfamida y prednisolona (protocolo COP) lograron $73 \%$ de remisión completa con un tiempo de supervivencia de 150 días, y adicionando Lasparaginasa el tiempo de supervivencia alcanzó a 210 días. En ese estudio se utilizaron protocolos de quimioterapia multicíclicos, indicando que los resultados dependen del lugar anatómico de la patología y del estatus de ViLeF del paciente. Asimismo, Teske (2002) trabajó con el protocolo COP, el protocolo VCM (vincristina, ciclofosfamida y metotrexato) y VCM más L-Asparaginasa logrando diversos grados de éxito; mientras que Ettinger (2003) con la doxorrubicina como único agente quimioterapéutico y con el protocolo COP más doxorrubicina consiguió la remisión completa de $26-32 \%$ y $47-54 \%$ de los casos, respectivamente. Simon et al. (2008) y Hadden et al. (2008) aplicaron igualmente diversos tratamientos cuyos resultados dependieron del estado del paciente en el momento de la terapia. 
De todos los antineoplásicos mencionados, la L-asparaginasa es un agente relativamente nuevo para el tratamiento del linfoma felino, habiendo más evidencias de su uso en caninos (LeBlanc et al., 2007). La Lasparagina es un aminoácido no esencial de los mamíferos, de modo que la Lasparaginasa disminuye a las células de asparagina, ocasionando una disminución de la síntesis proteica y muerte celular en aquellas células que no tienen capacidad de sintetizarla, como es el caso de las neoplasias linforeticulares (Plumb, 2010).

Con respecto a los efectos secundarios en los pacientes, se reporta toxicidad gastrointestinal leve a moderada con vincristina, doxorubicina, metotrexato y ciclofosfamida, que puede ser controlada (Simon et al., 2008). La toxicosis hemática también fue reportada con vincristina, pero se revierte en la mayoría de los casos el grado de neutropenia que ocasiona (Plumb, 2010).

En el Perú no es común realizar quimioterapias antineoplásicas en felinos, por lo que se comparte esta experiencia con la finalidad de comunicar los resultados del protocolo utilizado.

\section{Caso Clínico}

Se atendió en la Clínica Veterinaria Docente Cayetano Heredia de la Facultad de Medicina Veterinaria y Zootecnia de la Universidad Peruana Cayetano Heredia (Lima, Perú) un espécimen felino doméstico común americano, macho, de dos años, castrado, con $6.80 \mathrm{~kg}$, sin vacunas ni desparasitación al día, proveniente de la ciudad de Lima. El paciente fue adoptado hace un año y convivió con otros cinco gatos. Al examen clínico, el animal presentó una temperatura de $38^{\circ} \mathrm{C}$, disnea inspiratoria restrictiva superficial, tiempo de llenado capilar $<2 \mathrm{~s}$, frecuencia cardiaca de 140 latidos por minuto, frecuencia respiratoria de 62 por minuto.
El paciente fue internado y sometido a oxigenoterapia, tomándose muestras para analítica sanguínea, urianálisis y descarte de los virus de la inmunodeficiencia y leucemia felina mediante inmunocromatografia; asimismo, se tomaron placas de rayos $\mathrm{X}$ de la región torácica y una ecografía abdominal y torácica.

Los resultados del hemograma mostraron leucopenia $(3200 / \mu 1)$, linfopenia $(512 / \mu 1)$ y recuento plaquetario normal. La serología solo detectó antígeno de ViLeF, en tanto que el estudio bioquímico y el urianálisis no revelaron anormalidades. En las imágenes radiográficas de tórax, en vista lateral se observó incremento de radio opacidad en región cráneo ventral pulmonar, patrón bronquial en región dorso-caudal pulmonar y silueta cardiaca sin alteraciones; en tanto que en la vista ventro-dorsal se observó incremento de radio opacidad de ambos campos pulmonares, no distinguiéndose la silueta cardiaca (Figura 1).

En la ecografía torácica se observó líquido libre compatible con efusión pleural moderada, y en la zona craneal de tórax se observó una estructura redondeada de bordes irregulares e hipoecoico de 3.04 x 2.44 $\mathrm{cm}$, que sometida al modo doppler color mostró moderada vascularización (Figura 2A). Estas lesiones fueron compatibles con linfonódulo mediastinal neoplásico, no observándose anormalidades en el estudio ecocardiográfico. Adicionalmente, se realizó una toracocentesis para la obtención de muestra de la efusión torácica, la que al análisis citoquímico correspondió a un quilotórax. Una biopsia por punción con aguja fina del nódulo torácico para estudio citopatológico reveló presencia de células linfáticas atípicas correspondientes a un linfoma (Figura 2B).

Para mejorar la condición clínica del paciente se procedió a una toracocentesis, previa sedación con midazolam a $0.1 \mathrm{mg} / \mathrm{kg}$ y tramadol a $1 \mathrm{mg} / \mathrm{kg}$, ambos por vía IM. El paciente mostró mejoría luego de drenar el líquido, se restableció el apetito y mostró un 


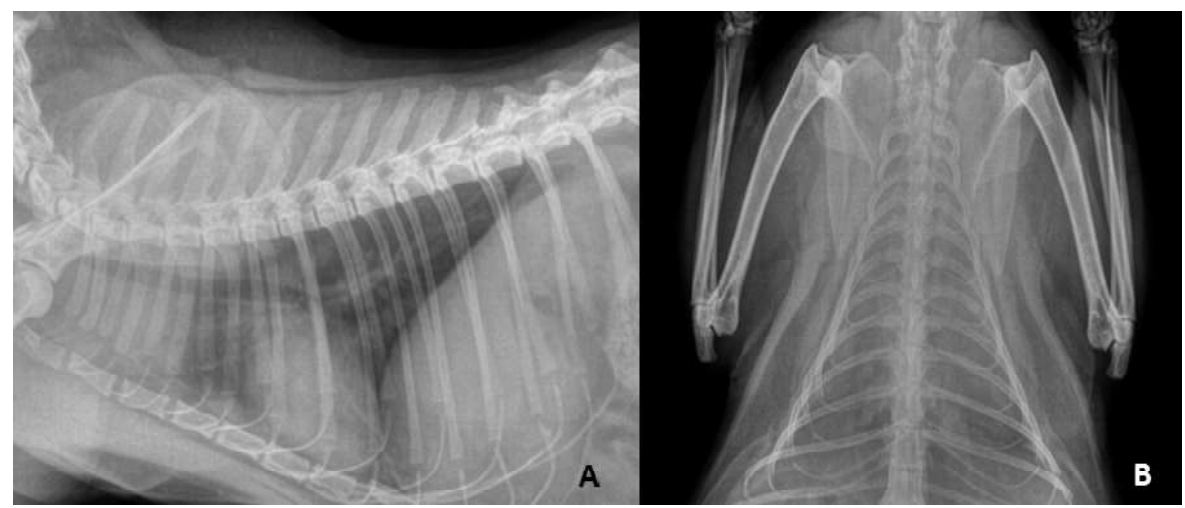

Figura 1. Imágenes radiográficas de paciente felino afectado con linfoma mediastinal. A, vista lateral derecha de tórax donde se observa incremento de radio opacidad en región cráneo ventral pulmonar, patrón bronquial en región dorso caudal pulmonar y silueta cardiaca sin alteraciones. $\mathrm{B}$, en la vista ventro dorsal de tórax se observa incremento de radio opacidad de ambos campos pulmonares, no distinguiéndose la silueta cardiaca

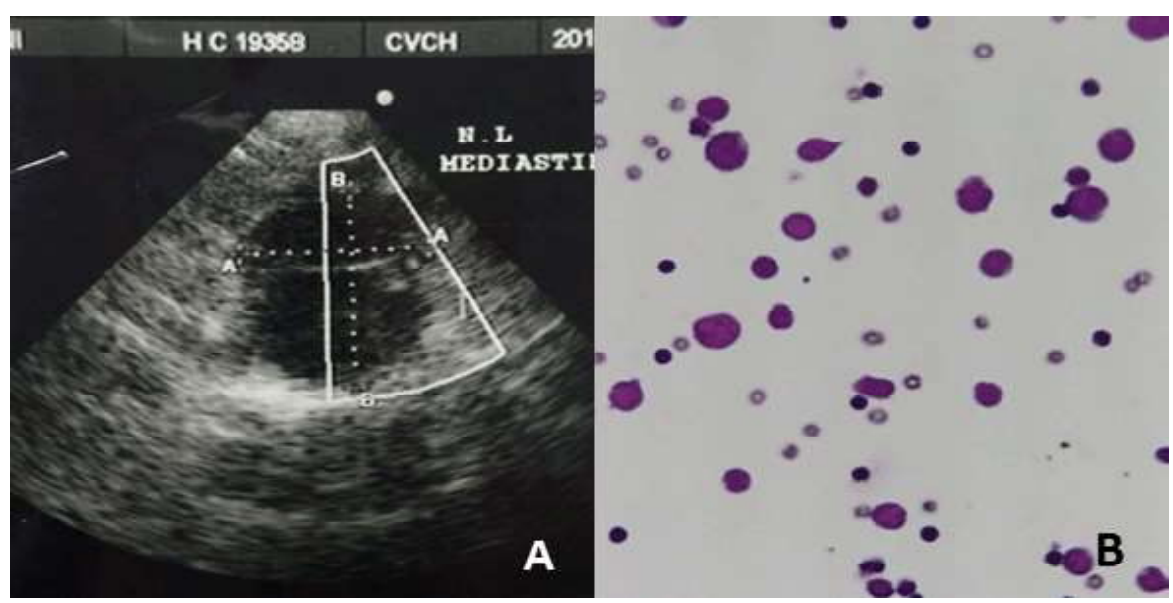

Figura 2. A, imagen ecográfica de tórax de paciente felino donde se observa estructura redondeada hipoecogénica de $3.04 \times 2.44 \mathrm{~cm}$, que muestra moderada vascularización al modo doppler color, compatible con linfonódulo mediastinal neoplásico. $\mathrm{B}$, imagen citológica obtenida mediante punción eco-guiada de linfonódulo mostrando células linfáticas atípicas correspondientes a linfoma (tinción Hemacolor 400x)

mejor ánimo. Se le mantuvo con tratamiento conservativo con amoxicilina más ácido clavulánico $\mathrm{PO}$ a $15 \mathrm{mg} / \mathrm{kg}$ y prednisolona PO a $5 \mathrm{mg}$ dosis total cada $12 \mathrm{~h}$, hasta que los dueños decidieran si se realizaría la quimioterapia.
Luego de 10 días el paciente presentó un cuadro de insuficiencia respiratoria marcada, motivo por el cual los propietarios autorizaron el inicio de la quimioterapia (Cuadro 1), previos análisis hematológicos. En la primera semana se inició la fase de induc- 
Cuadro 1. Protocolo de quimioterapia empleado en felino con linfoma mediastinal. Incluyó el uso de L-asparaginasa, vincristina, ciclofosfamida, prednisolona y doxorrubicina

\begin{tabular}{lcccccccccc}
\hline Semanas & 1 & 2 & 3 & 4 & 5 & 6 & 7 & 8 & 9 & 10 \\
\hline Sesiones/ quimioterápicas & $1^{\text {ra }}$ & $2^{\text {da }}$ & $*$ & $3^{\text {ra }}$ & $*$ & $4^{\text {ta }}$ & $5^{\text {ta }}$ & $*$ & $6^{\text {ta }}$ & $7^{\text {ma }}$ \\
\hline L-asparaginasa, $400 \mathrm{UI} / \mathrm{kg} \mathrm{SC}$ & $\mathrm{v}$ & - & - & - & - & - & $\mathrm{v}$ & - & - & - \\
Vincristina, $0.1 \mathrm{mg}$ EV & $\mathrm{v}$ & $\mathrm{v}$ & - & $\mathrm{v}$ & - & - & $\mathrm{v}$ & - & $\mathrm{v}$ & $\mathrm{v}$ \\
Ciclofosfamida, $150 \mathrm{mg} / \mathrm{m} 2 \mathrm{EV}$ & - & $\mathrm{v}$ & - & $\mathrm{v}$ & - & - & - & - & $\mathrm{v}$ & $*$ \\
Doxorrubicina, $1 \mathrm{mg} / \mathrm{kg} \mathrm{EV}$ & - & - & - & - & - & $\mathrm{v}$ & - & - & - & - \\
Prednisolona, $5 \mathrm{mg}$ dosis total PO & $\mathrm{v}$ & $\mathrm{v}$ & $\mathrm{v}$ & $\mathrm{v}$ & $\mathrm{v}$ & $\mathrm{v}$ & $\mathrm{v}$ & $\mathrm{v}$ & $\mathrm{v}$ & $\mathrm{v}$ \\
\hline
\end{tabular}

* No recibió medicación porque presentó efectos secundarios

ción con L-asparaginasa a $400 \mathrm{UI} / \mathrm{kg} \mathrm{SC}$ y vincristina a $0.1 \mathrm{mg} / \mathrm{kg} \mathrm{EV}$, observándose buen apetito y mejor ánimo; sin embargo, redujo su peso (de 6.4 a $5.8 \mathrm{~kg}$ ). Se realizó monitoreo mediante hemograma, y se procedió a la segunda sesión a la siguiente semana con vincristina a la misma dosis y ciclofosfamida a $150 \mathrm{mg} / \mathrm{m}^{2} \mathrm{EV}$. En la tercera semana, se suspendió la quimioterapia porque el hemograma evidenció neutropenia, manteniendo al paciente el tratamiento con amoxicilina más ácido clavulánico y prednisolona. El paciente mejoró en ánimo, apetito y comportamiento.

En la tercera sesión (cuarta semana), se repitió el esquema terapéutico de la segunda sesión, donde el paciente mantuvo el peso, buen ánimo y apetito. El tratamiento se suspendió la quinta semana, porque el hemograma mostró leucopenia y neutropenia, pero se le medicó amoxicilina más ácido clavulánico. En la cuarta sesión (sexta semana) se aplicó doxorrubicina a $1 \mathrm{mg} / \mathrm{kg} \mathrm{EV}$, $\mathrm{y}$ se le tomó una placa de rayos $\mathrm{X}$ de control, donde no se observaron alteraciones. El paciente siguió mostrando mejoría, se repitieron los análisis, y se procedió a la quinta sesión (séptima semana), repitiéndose el esque- ma de la primera semana. En el monitoreo se observó leucopenia por lo que se descansó una semana, y se realizó la sexta quimioterapia (novena semana) con vincristina y ciclofosfamida. En todo el proceso se continuó la terapia con prednisolona.

La séptima quimioterapia (décima semana) fue vincristina sin aplicar la ciclofosfamida. El paciente mostró decaimiento y vómitos después de la quimioterapia, pero luego se observó mejoría. Dos semanas después de la última sesión, la ecografía torácica de control mostró escasa a moderada cantidad de líquido libre, además de dos estructuras radio opacas pequeñas de 0.64 a $0.66 \mathrm{~cm}$, de ecogenicidad media, homogéneas adyacente a la silueta cardiaca. Se le hizo una terapia de mantenimiento con fluidos, antibióticos y antieméticos, así como toracocentesis para el control de líquido libre. En el hemograma y bioquímica control se observó leucocitosis (18 300/ $\mu 1)$.

Se evaluó la evolución del paciente y recomendó discontinuar el tratamiento debido a que no se logró la remisión total del linfoma, además del incremento de los efectos secundarios que se observaron durante la 
aplicación del mismo. El paciente se mantuvo con tratamiento sintomático, optándose por la eutanasia después de la última quimioterapia.

\section{Discusión}

El virus de la leucemia felina se transmite horizontalmente entre los gatos domésticos en condiciones naturales, especialmente en gatos jóvenes (Fujino et al., 2008), siendo los gatos infectados más propensos a desarrollar linfoma que aquellos que no lo están (Krunic et al., 2015). En el presente caso, correspondió a un gato joven infectado con ViLeF, con posibilidad que lo haya contraído antes que el dueño lo adoptara, al tener contacto con otros gatos infectados.

El linfoma es la neoplasia más común de origen hematopoyético en perros; sin embargo, no se origina por infección con retrovirus como sucede con los gatos y posee una incidencia menor que en los felinos (Louwerens et al., 2005). Los factores genéticos pueden tener un rol muy importante en la presentación de linfoma en gatos, siendo la raza Siamés donde el linfoma tiene mayor predilección por la ubicación mediastínica, y presentándose en animales en animales ViLeF negativos, por lo que se presume que existe un factor de mutación genética heredada que determina esta condición (Taylor y Harvey, 2015).

Los nódulos linfáticos caninos y felinos se encuentran usualmente elongados y con ecotextura homogénea e hipoecoicos con relación a la grasa adyacente (Schreurs et al., 2008); aspecto que fue observado en el presente caso en los ganglios linfáticos del mediastino. La ecografía junto con la radiología permitió monitorear la evolución de la enfermedad y la respuesta al tratamiento al poder obtener las dimensiones de los nódulos y la cantidad de efusión torácica; y correlacionarlo junto con el examen clínico y hematológico correspondiente. Por otro lado, la muestra obtenida con la punción con aguja fina y la citología correspondiente permitió realizar el diagnóstico de la enfermedad, como lo describen Louwerens et al. (2005).

El pronóstico del linfoma depende de su ubicación, siendo los más comunes y de peor pronóstico el gastrointestinal y el mediastínico (Sato et al., 2014). El presente caso correspondió a un linfoma mediastínico asociado a positividad al ViLeF y, debido a ello, de mal pronóstico, dado que los animales positivos a esta enfermedad tienen baja respuesta a la terapia y la respuesta depende sobre todo del estado inmunitario del paciente (Simon et al., 2008).

Teske et al. (2002) ha descrito que los gatos con linfoma mediastínico, sin recibir tratamiento, pueden fallecer en un periodo de pocos días a un máximo de 28 días, pudiendo la quimioterapia ofrecer una mejoría parcial y en pocos casos un rango de remisión completa del 92\%. En el presente caso, después de evaluar la respuesta a la toracocentesis y a la administración de prednisolona se recomendó la instauración de la quimioterapia, tratamiento que los propietarios no optaron en primera intención. Esta decisión no es fácil, ya que implica un gasto económico y mayor cuidado de la mascota, ya que pueden presentar efectos secundarios a la quimioterapia, como se observó en este caso.

No se observaron efectos secundarios durante la administración de L-asparaginasa, mostrando el gato buen apetito y ánimo. Las complicaciones posibles de la quimioterapia son la toxicidad no específica, hematológica y gastrointestinal que se presentan con el uso de la mayoría de las drogas antineoplásicas, con excepción de la bleomicina y Lasparaginasa, que se ha descrito que pueden presentar una reacción anafiláctica, vómitos, urticaria y mielosupresión si estas se administran junto con vincristina (Moore y Frimberger, 2009). La primera sesión de quimioterapia consistió en administrarle Lasparaginasa junto con vincristina, y por este motivo se realizó un seguimiento y monitoreo hematológico y clínico al paciente. 
La vincristina es un antineoplásico derivado de la vinca, usado para tratar principalmente neoplasias linfáticas y hematopoyéticas; y para su uso en el caso del linfoma felino se requiere un recuento de neutrófilos superior a 4500 células $/ \mu 1$, con pacientes bien hidratados antes de iniciar el tratamiento, y como recomendación de continuarse durante 24-36 h (Plumb, 2010). El paciente fue monitoreado y recibió fluidoterapia previa por $10 \mathrm{~h}$ continuas después de la quimioterapia; sin embargo, el paciente solo continuó un segundo ciclo incompleto de inducción de la quimioterapia debido que presentó signos de toxicidad.

Con respecto a la dosis, los protocolos recomiendan usar dosis de vincristina de 0.75 $\mathrm{mg} / \mathrm{m}^{2}$ (Teske et al., 2002). En este caso, y de acuerdo al protocolo, la dosis total indicada fue de $0.1 \mathrm{mg} E V$, ya que se trataba de un paciente con leucemia felina positivo y el riesgo de inmunosupresión era mayor. Cabe anotar que no existe una terapia «gold standard» para tratar linfoma felino (Collete et al., 2016).

Existen opciones terapéuticas limitadas para el tratamiento del virus de la leucemia felina. Se ha utilizado el 32 -azido-32 deoxitimidina (AZT; también conocido como zidovudina), pero se asocia a efectos secundarios significativos, que incluye manifestaciones aplásicas. Existen otros inmunomoduladores como el interferón alfa que es muy costoso y de eficacia limitada, y utilizada en conjunto con AZT aumenta el riesgo de toxicidad hematológica y hepática (Plumb, 2010). Debido al bajo conteo leucocitario del paciente y a la respuesta inicial a la quimioterapia, no se optó por realizar la terapia con estos fármacos.

El tiempo de supervivencia de este paciente fue de 112 días. Solo se observó signos de toxicidad en la sexta quimioterapia, lo cual limitó completar un segundo ciclo del esquema. En ese momento, se optó por hacerle una evaluación general, y se encontró nódulos en el mediastino, por lo que la remisión fue incompleta. Desde la detención del tratamiento hasta la muerte del animal se realizaron seguimientos ecográficos en donde se evidenció el incremento en la efusión, aumento de tamaño de los nódulos linfáticos y finalmente consolidación de segmentos pulmonares, por lo que el propietario optó por autorizar la eutanasia.

Debido a la localización de la neoplasia y al pobre estado inmunológico del paciente por la infección con ViLeF, se consideró que la respuesta al tratamiento fue baja; sin embargo, existieron periodos en los cuales se evidenció una mejoría clínica, por lo que se puede concluir que se logró alargar el tiempo y mejorar la calidad de vida del paciente con la quimioterapia instaurada.

\section{Literatura Citada}

1. Azakami D, Onozawa E, Miyabe M, Ochia K. Michishita M, Hira T, Momota Y, Ishioka K, Sako T. 2015. Primary T-cell high-grade lymphoma of the feline uterus. J Vet Med Sci 5: 913916. doi: 10.1292/jvms. 15-0575

2. Collete SA, Allstadt SD, Chon EM, Vernau W, Smith AN, Garrett LD, Choy K, et al. 2016. Treatment of feline intermediate to high-grade lymphoma with a modified university of WisconsinMadison protocol: 119 cases (20042012). Vet Comp Oncol 14: 136-146. doi: $10.1111 / \mathrm{vco} .12158$

3. Ettinger SN. 2003. Principles of treatment for feline lymphoma. Clin Tech Small Anim Pract 18: 98-102. doi: 10.1053/svms.2003.36623

4. Fujino Y, Ohno K, Tsujimoto H. 2008. Molecular pathogenesis of feline leukemia virus-induced malignancies: insertional mutagenesis. Vet Immunol Immunopathol 123: 138-143. doi: 10.1016/j.vetimm.2008.01.019 
5. Hadden G, Cotter SM, Rand W, Moore AS, Davis RM, Morrissey P. 2008. Efficacy and toxicosis of VELCAP-C treatment of lymphoma in cats. J Vet Intern Med 22: 153-157. doi: 10.1111/ j.1939-1676.2007.0031.x

6. Krunic M, Ertl R, Hagen B, Sedlazeck FJ, Hofmann-Lehmann $R$, von Haeseler A, Klein D. 2015. Decreased expression of endogenous feline leukemia virus in cat lymphomas: a case control study. BMC Vet Res 11: 90. doi: 10.1186/ s12917-015-0378-9

7. Kyoung-Won S, Soo Choi UI, Kyoung Bae B, Mi-Sun P, Cheol-Yong H, DaeYong K. 2006. Mediastinal lymphoma in a young Turkish Angora cat. J Vet Sci 2: 199-201. doi: 10.4142/jvs.2006.7.2.199

8. LeBlanc AK, Cox SK, Kirk CA, Newman SJ, Bartges JW, A.M. Legendre AM. 2007. Effects of 1asparaginase on plasma amino acid profiles and tumor burden in cats with lymphoma. J Vet Intern Med 21: 760-763.

9. Louwerens M, London CA, Pedersen NC, Lyons LA. 2005. Feline lymphoma in the post-feline leukemia virus era. J Vet Intern Med 19: 329-335. doi: 10.1111/ j.1939-1676.2005.tb02703.x

10. Moore AS, Cotter SM, Frimberger AE, Wood CA, Rand WM, L'Heureux DA. 1996. A comparison of doxorubicin and COP for maintenance of remission in cats with lymphoma. J Vet Intern Med 10: 372-375. doi: 10.1111/j.19391676.1996.tb02083.x

11. Moore A, Frimberger AE. 2009. Oncology for veterinarian technicians and nurses. Australia: Willey Blackwell. 318 p.
12. Plumb DC. 2010. Manual de farmacología veterinaria. $6^{\circ} \mathrm{ed}$. Argentina: Intermédica. $1256 \mathrm{p}$.

13. Sato H, Fujino Y, Chino J, Takahashi M, Fukushima K, Goto-Koshino Y, Kuchida K, et al. 2014. Prognostic analyses on anatomical and morphological classification of feline lymphoma. J Vet Med Sci 6: 807-811. doi: 10.1292/ jvms.13-0260

14. Schreurs E, Vermote K, Barberet V, Daminet S, Rudorf H, Saunders J.H. 2008. Ultrasonographic anatomy of abdominal lymph nodes in the normal cat. Vet Radiol Ultrasound 49: 68-72. doi: 10.1111/j.1740-8261.2007.00320.x

15. Simon D, Eberle N, Laacke-Singer L, Nolte I. 2008. Combination chemotherapy in feline lymphoma: treatment outcome, tolerability, and duration in 23 cats. J Vet Intern Med 22: 394-400. doi: 10.1111/j.1939-1676.2008.0057.x

16. Taylor S. Harvey A. 2015. Feline medicine. Review and test. UK: Saunders. $396 \mathrm{p}$.

17. Teske E, van Straten $G$, van Noort $R$, Rutteman GR. 2002. Chemotherapy with cyclophosphamide, vincristine, and prednisolone (COP) in cats with malignant lymphoma: new results with an old protocol. J Vet Intern 16: 179-186. doi: 10.1111/j.1939-1676.2002.tb02352.x

18. Vail DM, Moore AS, Ogilvie GK, Volk LM. 1998. Feline lymphoma (145 cases): proliferation indices, cluster of differentiation 3 immunoreactivity, and their association with prognosis in 90 cats. J Vet Intern Med 12: 349-354. doi: 10.1111/j.1939-1676.1998.tb02134.x 